safe space, somewhere they could openly share feelings of isolation, sadness, difference and frustration. Young people emphasised the need to normalise the way they felt and some focused on enhancing peers' self-esteem, confidence and the development of their own identity particularly in the context of support received from health professionals.

Conclusions Young people used the discussion groups to find a supportive peer community where they could share personal emotional and social experiences and obtain advice relating to living life with CF. This type of peer support is not replicable through self-care support and management advice delivered by health professionals; therefore online support groups have an important role in supplementing existing professional support.

\section{G23 YOUNG PEOPLE WITH DIABETES AND THEIR PEERS}

${ }^{1} \mathrm{JM}$ Brooks, ${ }^{2} \mathrm{~N}$ Kime, ${ }^{1} \mathrm{~N}$ King, ${ }^{3} \mathrm{~A}$ Wearden, ${ }^{1} \mathrm{~W}$ Gillibrand, ${ }^{4} \mathrm{~F}$ Campbell. ${ }^{1}$ Centre for Applied Psychological and Health Research, University of Huddersfield, Huddersfield, UK; ${ }^{2}$ Carnegie Faculty, Leeds Beckett University, Leeds, UK; ${ }^{3}$ School of Psychological Sciences, University of Manchester, Manchester, UK; ${ }^{4}$ Childrens Diabetes Unit, Leeds Taching Hospitals NHS Trust, Leeds, UK

\subsection{6/archdischild-2015-308599.23}

Aims Peer influences can impact across a range of adolescent behaviours. Type 1 diabetes (T1D) is amongst the most common forms of chronic illness to affect young people in the UK. Selfmanagement is crucial, but managing their T1D is often very difficult for adolescents. It has been suggested that interventions to support adolescents with T1D could usefully include friends and peers, but little is known about how peers think about and respond to T1D. This research explored T1D from the perspective of adolescent patients, friends and peers.

Methods We conducted dyadic interviews with adolescent T1D patients aged 13-15 years and a nominated close friend $(\mathrm{N}=20)$. We then carried out three focus group sessions with participants without any necessary prior T1D knowledge in a secondary school setting.

Data were thematically coded and final data interpretations were subject to independent scrutiny from young people the same age as our research participants, who assessed the findings in terms of relevance and usefulness from their own perspective. Results Limited awareness of T1D amongst their peer group generally and school teaching staff was reported by interview participants. Close friends play a valuable role in recognising the importance of T1D management whilst also accepting and normalising the condition. Considerable variations in experiences of support provided in relation to T1D in the school environment were reported by all participants, including the extent to which peers were encouraged or even permitted to support patients.

Educational materials designed in response to issues raised in the interviews were piloted in classroom settings. Realistic vignettes developed from the interviews were used to facilitate group discussion. The simple materials were well-received and positively evaluated.

Conclusions This research highlights the need for greater awareness of T1D in schools amongst teachers and peers, but encouragingly suggests that simple educational sessions can be easily implemented and are well-received. The Children and Families Act (2014) means that safe and effective care for young people with T1D must now by law be embedded in the school system. Diabetes specialist nurses should be aware of potential peer influences and may be able to usefully assist schools looking to sensitively and approriately raise T1D awareness and faciitate optimal peer support.

\section{G24 PATIENT EXPERIENCE: CAPTURING THE CHILD'S PERSPECTIVE}

J Higson. Paediatrics, Royal Berkshire Hospital, Reading, UK

\subsection{6/archdischild-2015-308599.24}

Aims The aim of this work was to capture the experiences of children and families during their admission to a district general hospital.

Methods This was a mixed methods study. Three different surveys were developed for use by parents and carers, children aged 11-16 years and children aged 6-10 years. To add depth and richness to the data children's stories, pictures and poems were collected to generate data that reflected the children's creativity and imagination. Data were collected between January 2013 and December 2013.

Results A total of 560 parent surveys were returned from the day care ward $(n=362)$ and the clinic $(n=198)$. A range of different factors were identified that could improve services. These can broadly be divided into two categories. Category1: Child and family centred factors included the following: some children aged 11-16 wanted the opportunity to speak to clinical staff without their parents present; there were not enough play facilities for children aged 11-16; not all staff were introducing themselves to the children or families; the environment could benefit from improvement. Category 2: concerns were raised about parents' expectations about waiting times in clinic were not always being managed; on the day care ward some family's were not kept informed of the delays for theatre or given realistic expectations for how the day will run.; and telephone follow up was not offered to all families.

The pictures and poems from children highlighted that food and play were the most important factors to them.

Conclusion Eliciting children's feedback on service provision is vital to make improvements to the services we provide. They are the ones experiencing our service and as clinicians we need to be engaging with them using different methods to ask them what we can do to improve the care we provide.

This work highlighted many areas for small changes that have led the staff to reflect on the services and adapt to meet the needs of the children.

\section{G25 COMMUNICATING WITH PARENTS FOLLOWING A SUSPECTED ADVERSE DRUG REACTION IN A CHILD: WHO SAYS WHAT AND WHEN?}

\begin{abstract}
${ }^{1,2}$ J Arnott, ${ }^{2}$ AJ Nunn, ${ }^{2}$ H Mannix, ${ }^{1,2}$ M Peak, ${ }^{3}$ M Pirmohamed, ${ }^{4,5}$ RL Smyth, ${ }^{3,6}$ MA Turner, ${ }^{7} B$ Young. ${ }^{1}$ School of Health, University of Central Lancashire, Preston, UK; ${ }^{2}$ Research and Development, Alder Hey Children's NHS Foundation Trust, Liverpool, UK; ${ }^{3}$ Institute of Translational Health, University of Liverpool, Liverpool, UK; ${ }^{4}$ Institute of Child Health, University College London, London, UK; ${ }^{5}$ Institute of Child Health, Great Ormond Street Hospital Foundation Trust, London, UK; ${ }^{6}$ Department of Women's and Children's Health, Liverpool Women's NHS Foundation Trust, Liverpool, UK; ${ }^{7}$ Institute of Psychology Health and Society, University of Liverpool, Liverpool, UK
\end{abstract}

\subsection{6/archdischild-2015-308599.25}

Aim To pilot a prompt guide to support discussions between clinicians and parents following a suspect adverse drug reaction in a child. 